\title{
Variety in fruit and vegetable intake and cognitive function in middle-aged and older Puerto Rican adults
}

\author{
Xingwang Ye, Shilpa N. Bhupathiraju and Katherine L. Tucker* \\ Department of Health Sciences, Northeastern University, 316 Robinson Hall, 360 Huntington Avenue, Boston, \\ MA 02115, USA \\ (Submitted 20 June 2011 - Final revision received 16 January 2012 - Accepted 1 March 2012 - First published online 1 May 2012)
}

\begin{abstract}
Higher variety in fruit and vegetable intake has been associated with a lower risk of several chronic diseases. It remains unclear whether such associations exist relating to cognition. The authors examined associations between total quantity and variety in fruit and vegetable intake and cognitive function in a cross-sectional sample of 1412 Puerto Rican adults, aged 45-75 years from the Boston Puerto Rican Health Study, 2004-9. Fruit and vegetable intake was assessed with a FFQ. Cognitive function was measured with a battery of seven tests; the Mini Mental State Examination (MMSE) was administrated to assess global cognitive function. Greater variety, but not total quantity, of fruit and vegetable intake was associated with a higher MMSE score after multivariate adjustment $(P$ for trend $=0 \cdot 012)$. This association remained significant after further adjusting for total quantity of fruit and vegetable intake ( $P$ for trend $=0 \cdot 018)$. High variety of fruit and vegetable intake was also associated with individual cognitive domains, including executive function, memory and attention (all $P$ for trend $<0.05$ ). Variety, more than total quantity, of fruit and vegetable intake may offer cognitive protection in middle-aged and older adults, but longitudinal studies are needed to clarify direction of causality.
\end{abstract}

Key words: Fruit: Vegetables: Dietary variety: Cognition: Middle-aged adults: Puerto Ricans

Greater fruit and/or vegetable intake has been associated with a lower risk of several chronic diseases, including $\mathrm{CHD}^{(1,2)}$, stroke $^{(3,4)}$ and several types of cancers ${ }^{(5-7)}$. A higher intake of vegetables has also been associated with less cognitive decline ${ }^{(8,9)}$; and a lower intake of fruit and vegetables has been related to poorer executive function and memory ${ }^{(10)}$ and a higher risk of dementia and Alzheimer's disease ${ }^{(11)}$.

Fruit and vegetables are generally rich in antioxidants (vitamin C, carotenoids, etc.) and other bioactive compounds. Dietary antioxidants have been associated with a lower risk of dementia and Alzheimer's disease in some studies ${ }^{(12,13)}$, but not others ${ }^{(14,15)}$. However, several intervention studies with antioxidant vitamins have not shown beneficial effects on cognition in older adults ${ }^{(16-19)}$. The inconsistency among these studies could be due to differences in study design, or to limitations in the supplements provided - in that other nutrients or bioactive compounds in fruit and vegetables, rather than investigated specific antioxidant vitamins, may be protective against cognitive decline. Further, different fruits and vegetables have varied nutrients and bioactive compounds. The combination of different types of fruits and vegetables may provide additive and/or synergistic beneficial effects relative to individual fruits and vegetables ${ }^{(20)}$.

To our knowledge, no studies have examined the association between variety in fruit and vegetable intake and cognitive function. We aimed to simultaneously study the associations between variety and total quantity of fruit and vegetable intake and cognitive function in a middle-aged and older population.

\section{Materials and methods}

\section{Participants}

Participants in the baseline interview of the Boston Puerto Rican Health Study, an ongoing study to explore sociological, environmental and genetic factors contributing to ageingrelated chronic diseases and quality of life, were included in these analyses ${ }^{(21)}$. Details of this study design have been reported previously ${ }^{(21)}$. In brief, baseline data collection was completed between 2004 and 2009. Census tracts with twenty-five or more Puerto Rican adults, aged 45-75 years, were selected from the year 2000 census in the greater Boston, Massachusetts area, and all blocks with ten or more

Abbreviations: ADL, Activities of Daily Living; MMSE, Mini Mental State Examination.

*Corresponding author: K. L. Tucker, fax +1 617373 2968, email kl.tucker@neu.edu 
Hispanic adults, aged 45-75 years, were enumerated. If more than one household member qualified for the study, one was randomly selected. Approximately $77.4 \%$ of our sample was identified with this method, with additional participants solicited randomly at major community events $(9 \cdot 8 \%)$, from referral $(7 \cdot 2 \%)$ or individual calls to the study (5.6\%). Of the 2170 individuals identified, seventy-seven were excluded because of serious health conditions that would preclude answering questions, a planned move away within 2 years or a lack of a permanent address. Of those remaining, 1811 agreed to participate and 1500 (83.2\%) completed the baseline survey: 302 participants did not finish the survey due to difficulty in scheduling or unsuccessful followup; and nine were excluded because of their low Mini Mental State Examination (MMSE) scores $(\leq 10$, indicating severe cognitive impairment) ${ }^{(22)}$. For the present analysis, we further excluded individuals with implausible energy intake $(<2510 \mathrm{~kJ} / \mathrm{d}(600 \mathrm{kcal} / \mathrm{d})$ or $>20083 \mathrm{~kJ} / \mathrm{d}(4800 \mathrm{kcal} / \mathrm{d}))$ and those with incomplete cognitive tests or blood lipids, resulting in a sample of 1412 . The present study was conducted according to the guidelines laid down in the Declaration of Helsinki and all procedures involving human subjects were approved by the Institutional Review Board at Tufts Medical Center. Written informed consent was obtained from all subjects.

\section{Variety and quantity of fruit and vegetable intake}

Information about dietary intake was collected for the previous 12 months with a validated semi-quantitative FFQ with 223 items, interviewer-administered in the home ${ }^{(21)}$. Nutrient intake was calculated using the Nutrition Data System for Research software version 2007 (Nutrition Coordinating Center, University of Minnesota, Minneapolis, MN, USA, Food and Nutrient Database 2007). The number of servings of fruit and vegetables per $d$ was calculated using reference serving sizes from the Pyramid Servings Database for USDA Survey Food Codes version $2.0^{(23)}$. The FFQ includes questions for the intake of specific fruits, vegetables, $100 \%$ fruit juice and $100 \%$ vegetable juice ${ }^{(24)}$. Dried beans and starchy vegetables (potatoes and potato products, plantains, tannier and cassava) were not included for the calculation of total quantity and variety in fruit and vegetable intake. Each specific type of fruit, vegetable, $100 \%$ fruit juice or $100 \%$ vegetable juice that was consumed at least once per month was counted towards the variety score. The possible ranges for the summed variety scores were from 0 to 27 for fruit intake (including $100 \%$ fruit juice) and from 0 to 26 for vegetable intake (including $100 \%$ vegetable juice). The FFQ fruit and vegetable items are shown in Table S1 (available online).

\section{Assessment of cognitive function}

A trained research assistant administered a battery of seven neuropsychological tests with each participant, in his or her home, before the FFQ data were collected. The MMSE was used as a measure of global cognitive function (ranged from 12 to 30 in the present sample) ${ }^{(25)}$. Other cognitive tests included: a word-list learning test to assess verbal memory, with sub-scores for learning and immediate recall, recognition, and percentage retention ${ }^{(26)}$; digit span forward and backward tests to measure attention and working memory ${ }^{(26)}$; the Stroop test for cognitive flexibility, response inhibition and processing speed ${ }^{(21)}$; the verbal fluency test to measure the speed at which one can provide examples to a category (initial letter of a word) ${ }^{(26)}$; clock-drawing ${ }^{(27)}$ and figure-copying ${ }^{(28)}$ tests to measure visual-spatial organisation. Scores for figure copying were weighted for the complexity of the figure, by assigning 1 point for easy figures and to 4 points for the most difficult. Greater scores on each of these seven tests reflect better cognition.

The MMSE score was our primary outcome because the MMSE measures global cognitive function. Scores from each neuropsychological test were standardised with a mean of 0 and a standard deviation of 1 . A factor analysis for data reduction was then conducted with standardised scores. After performing varimax rotation, three meaningful cognitive function factors were obtained: executive function; memory; attention (Table S2, available online). The standardised factor-scoring coefficients for converting individual test scores to factor scores are shown in Table S3 (available online).

\section{Assessment of covariates}

Educational attainment was categorised into four categories: none or primary school; secondary school; high school; college or higher. Participants were classified as never smoker $(<100$ cigarettes in entire life), former smoker or current smoker. Alcohol use was classified as not current, current moderate ( $\leq 1 \mathrm{drink} / \mathrm{d}$ for women or $\leq 2 \mathrm{drinks} / \mathrm{d}$ for men) or current heavy $(>1 \mathrm{drink} / \mathrm{d}$ for women or $>2 \mathrm{drinks} / \mathrm{d}$ for men). Physical activity was evaluated with a modified Paffenbarger questionnaire from the Harvard Alumni Activity Survey ${ }^{(21)}$. Poverty was defined 'yes' if a participant's total annual household income was below the poverty threshold released each year by the US Department of Health and Human Services, relative to family size. For example, the poverty threshold for a family with two members was $\$ 13690$ for Massachusetts residents in $2007^{(29)}$. An acculturation score was calculated based on answers to seven questions regarding the extent of use of English and/or Spanish for work and daily life ${ }^{(21)}$, ranging from 0 (only using Spanish) to 100 (only using English). Physical disability was assessed with a modified Activities of Daily Living (ADL) scale with twelve items with total scores ranging from 0 to $36^{(30)}$. Greater ADL scores indicate lower physical function.

During the home interview, height and weight were measured with standard methods ${ }^{(21)}$, and BMI was calculated. Blood pressure was measured at three time points during the home interview with the latter two measures averaged for data analysis. Hypertension was defined as systolic blood pressure $\geq 140 \mathrm{mmHg}$ or diastolic blood pressure $\geq 90 \mathrm{mmHg}$, or use of antihypertensive medications. Fasting blood samples were collected and concentrations of glucose, total cholesterol, HDL-cholesterol and TAG were determined with standardised 
methods ${ }^{(21)}$. Diabetes was defined as 'yes' if a participant had fasting glucose $\geq 7 \cdot 0 \mathrm{mmol} / 1$ or was taking medication for diabetes ${ }^{(31)}$.

\section{Statistical analyses}

Participants were placed in quintiles according to the total number of servings and variety score of fruit and vegetable intake, respectively. General linear models were used to compare continuous variables and logistic regression models were used to compare categorical outcome variables across quintiles of total intake quantity and variety scores. Means in the last four quintiles were compared with means in the first quintile, after Tukey's adjustment for multiple comparisons. Cognitive performance scores for each quintile were calculated after multivariate adjustment with general linear models. In the first model, we adjusted for age (years), sex, educational attainment (none or primary school, secondary school, high school, or college or higher), income below the poverty threshold (yes/no), acculturation score (continuous), BMI $\left(\mathrm{kg} / \mathrm{m}^{2}\right)$ and total energy intake $(\mathrm{kJ} / \mathrm{d})$. In a second model, we further adjusted for potential confounders and mediators, including smoking status (never, past smoker or current smoker), alcohol use (never, moderate or high), physical activity score (continuous), supplement use (yes/no), use of five or more types of medications within the past 12 months (yes/no), ADL score (continuous), hypertension (yes/no) and diabetes (yes/no). In a final full model, we further adjusted for the variety score for the total serving variable, and vice versa. Original continuous total servings and variety score were used for trend tests. We also investigated whether lipids (total cholesterol, HDL-cholesterol and TAG) or the dietary intake of PUFA and MUFA would attenuate the associations between total servings and variety score and cognitive function. In a secondary analysis, we repeated all analyses to examine whether fruit intake (servings $v$. variety) or vegetable intake (servings $v$. variety) was individually associated with cognitive function. All statistical analyses were conducted with SAS version 9.2 (SAS Institute, Inc.). A value of $P<0.05$ (two-sided) was considered statistically significant.

\section{Results}

Participants in the highest quintile of fruit and vegetable intake had a 4.6-fold higher intake than those in the first quintile (median 6.5 v. 1.4 servings/d; Table 1), and those in the highest quintile of variety in fruit and vegetable intake consumed twice the variety of those in the lowest quintile. Those with higher fruit and vegetable intake, or with higher fruit and vegetable variety, tended to have education at the college level or higher, to be not current smokers, to drink alcohol moderately, to have a higher physical activity score, a lower ADL score, and to have a higher intake of total energy and a higher use of supplements, relative to those with the lowest quantity or variety in fruit and vegetable intake, respectively. Participants with the highest variety scores also tended to be female, to be more acculturated, above the poverty line, to have higher total and HDL-cholesterol concentrations, and were less likely to have diabetes and to use medications, than those with the lowest variety scores. The total quantity of fruit and vegetable intake was moderately associated with the variety score (Spearman's correlation coefficient $0 \cdot 34, P<0 \cdot 001)$.

Higher variety in fruit and vegetable intake ( $P$ for trend $<0 \cdot 001$ ), but not quantity ( $P$ for trend $=0 \cdot 13$ ), was associated with a greater MMSE score after adjustment for age, sex, educational attainment, household income, acculturation score, BMI and total energy intake (model 1; Table 2). The association between variety in fruit and vegetable intake and the MMSE score remained significant after further adjustment for smoking, alcohol use, physical activity score, supplement use, medication use, ADL score, hypertension and diabetes (model 2, $P$ for trend=0.012), and total quantity of fruit and vegetable intake (model 3, $P$ for trend=0.018). Each of the seven points of the fruit and vegetable variety score ( $\beta=0 \cdot 17$, SE $=0 \cdot 07, P=0 \cdot 012)$ was also inversely similar to 5 years of age on MMSE scores $(\beta=-0 \cdot 16$, SE $=0 \cdot 07$, $P=0 \cdot 018$ ), after multivariate adjustment in model 3. Higher variety score was also associated with better executive function, memory and attention (model $1-3$, all $P$ values for trend $<0.05$ ). Further adjustment for blood lipids (total cholesterol, HDL-cholesterol and TAG) and dietary intake of PUFA and MUFA did not change the observed associations mentioned above (data not shown).

We further examined the associations between total quantity and variety in fruit and vegetable intake and the scores of individual cognitive tests (Table 3). A higher fruit and vegetable variety score was significantly associated with better scores for word-list immediate recall, word-list percentage recognition and retention, letter fluency, digit span forward and backward, and clock drawing, after multivariate adjustment including total intake of fruits and vegetables (all $P$ values for trend $<0 \cdot 05)$.

In secondary analyses, higher variety, but not quantity, of fruit intake was significantly associated with a higher MMSE score, and with the executive function, memory and attention factors, after multivariate adjustment (model $1-3$, all $P$ values for trend $<0.05$; Table S4, available online). Higher quantity and variety in vegetable intake were both significantly associated with a greater MMSE score after adjustment for age, sex, education, household income below the threshold, acculturation score, BMI and total energy intake ( $P$ for trend $<0.05$; Table S5, available online). Variety, but not quantity, of vegetable intake remained significantly associated with the MMSE score after further adjustment for lifestyle factors, supplement and medication uses, ADL score, diabetes and hypertension ( $P$ for trend $=0 \cdot 024$ ). Higher variety in vegetable intake was marginally significantly associated with the MMSE score ( $P$ for trend $=0.065$ ) and memory $(P$ for trend $=0.067)$, and significantly associated with executive function and attention (both $P$ for trend $<0.05$ ) after further adjustment for total quantity of vegetable intake. 
Table 1. Characteristics of the participants by quintiles of total quantity and variety in fruit and vegetable intake from the Boston Puerto Rican Health Study, 2004-9 (Mean values with their standard errors; number of participants; percentages; medians and ranges)

\begin{tabular}{|c|c|c|c|c|c|c|c|c|c|c|c|c|c|c|}
\hline \multirow[b]{3}{*}{ Characteristics } & \multicolumn{6}{|c|}{ Fruit and vegetable intake (servings/d) } & \multirow[b]{3}{*}{ P† } & \multicolumn{6}{|c|}{ Fruit and vegetable variety } & \multirow[b]{3}{*}{$P+$} \\
\hline & \multicolumn{2}{|c|}{ Quintile 1} & \multicolumn{2}{|c|}{ Quintile 3} & \multicolumn{2}{|c|}{ Quintile 5} & & \multicolumn{2}{|c|}{ Quintile 1} & \multicolumn{2}{|c|}{ Quintile 3} & \multicolumn{2}{|c|}{ Quintile 5} & \\
\hline & Mean & SE & Mean & SE & Mean & SE & & Mean & SE & Mean & SE & Mean & SE & \\
\hline$n$ & \multicolumn{2}{|c|}{282} & \multicolumn{2}{|c|}{282} & \multicolumn{2}{|c|}{282} & & \multicolumn{2}{|c|}{276} & \multicolumn{2}{|c|}{283} & \multicolumn{2}{|c|}{286} & \\
\hline Median & \multicolumn{2}{|c|}{1.4} & \multirow{2}{*}{\multicolumn{2}{|c|}{$\begin{array}{c}3.2 \\
2.8-3.7\end{array}$}} & \multirow{2}{*}{\multicolumn{2}{|c|}{$\begin{array}{c}6 \cdot 5 \\
5 \cdot 2-16 \cdot 8\end{array}$}} & & \multirow{2}{*}{\multicolumn{2}{|c|}{$\begin{array}{c}19 \\
2-23\end{array}$}} & \multirow{2}{*}{\multicolumn{2}{|c|}{$\begin{array}{c}32 \\
30-34\end{array}$}} & \multirow{2}{*}{\multicolumn{2}{|c|}{$\begin{array}{c}44 \\
40-52\end{array}$}} & \\
\hline Range & \multicolumn{2}{|c|}{$0.2-1.9$} & & & & & & & & & & & & \\
\hline Intake, variety & 1.3 & 0.4 & $3 \cdot 2$ & 0.3 & $7 \cdot 0$ & $1 \cdot 8$ & & $18 \cdot 0$ & 4.4 & $32 \cdot 1$ & 1.4 & $44 \cdot 2$ & 3.3 & \\
\hline Age (years) & $57 \cdot 2$ & $8 \cdot 0$ & $57 \cdot 0$ & $7 \cdot 7$ & 58.0 & 7.4 & 0.31 & $56 \cdot 1$ & $7 \cdot 6$ & $57 \cdot 3$ & 7.8 & $56 \cdot 9$ & $7 \cdot 0$ & 0.11 \\
\hline Female (\%) & & & & & & & 0.12 & & & & & & & 0.003 \\
\hline Educational attainment (\%) & & & & & & & 0.003 & & & & & & & $<0.001$ \\
\hline None or primary school & & & & & & & & & & & & & & \\
\hline Secondary school & & & & & & & & & & & & & & \\
\hline High school & & & & & & & & & & & & & & \\
\hline College or higher & & & & & & & & & & & & & & \\
\hline Household income below the threshold (\%) & & & & & & & 0.57 & & & & & & & $<0.001$ \\
\hline Acculturation score & 22.9 & $22 \cdot 3$ & 24.5 & 23.4 & $26 \cdot 1$ & $22 \cdot 3$ & 0.53 & $21 \cdot 0$ & $22 \cdot 8$ & 22.4 & $22 \cdot 0$ & $32 \cdot 8^{\star \star \star}$ & $23 \cdot 3$ & $<0.001$ \\
\hline Smoking status (\%) & & & & & & & 0.024 & & & & & & & 0.004 \\
\hline Never & & & & & & & & & & & & & & \\
\hline Past smoker & & & & & & & & & & & & & & \\
\hline Current smoker & & & & & & & & & & & & & & \\
\hline Alcohol use (\%) & & & & & & & 0.002 & & & & & & & $<0.001$ \\
\hline Never & & & & & & & & & & & & & & \\
\hline Moderate & & & & & & & & & & & & & & \\
\hline High & & & & & & & & & & & & & & \\
\hline Physical activity score & $30 \cdot 6$ & 4.9 & $31 \cdot 3^{\star \star}$ & 3.8 & $32 \cdot 1^{\star \star}$ & 4.9 & 0.002 & 31.3 & 4.9 & 31.0 & 4.4 & $32 \cdot 5^{\star}$ & $5 \cdot 0$ & $<0.001$ \\
\hline ADL score & 4.9 & $6 \cdot 2$ & $4 \cdot 1$ & $5 \cdot 1$ & 4.0 & 4.7 & 0.039 & 4.6 & 5.9 & 4.8 & 5.4 & $3.0^{*}$ & $4 \cdot 1$ & $<0.001$ \\
\hline Energy intake $(\mathrm{kJ} / \mathrm{d})$ & 6607 & 2874 & $8791^{\star \star \star}$ & 3305 & $11473^{\star \star \star}$ & 916 & $<0.001$ & 8272 & 3502 & 9021 & 3774 & $9435^{\star \star}$ & 3736 & 0.004 \\
\hline Hypertension (\%) & & & & & & & 0.93 & & & & & & & 0.58 \\
\hline Diabetes (\%) & & & & & & & 0.36 & & & & & & & 0.003 \\
\hline BMI $\left(\mathrm{kg} / \mathrm{m}^{2}\right)$ & $31 \cdot 7$ & $7 \cdot 0$ & $31 \cdot 6$ & 6.6 & $32 \cdot 6$ & $6 \cdot 6$ & 0.21 & $31 \cdot 0$ & $6 \cdot 7$ & $32 \cdot 4$ & $6 \cdot 4$ & 31.7 & 6.5 & 0.039 \\
\hline Total cholesterol $(\mathrm{mmol} / \mathrm{l})$ & 4.67 & $1 \cdot 11$ & 4.80 & 1.03 & 4.74 & 1.10 & 0.69 & 4.62 & 1.11 & 4.69 & 1.09 & 4.82 & 1.03 & 0.049 \\
\hline HDL-cholesterol (mmol/l) & 1.17 & 0.36 & $1 \cdot 15$ & 0.31 & $1 \cdot 17$ & 0.34 & 0.61 & 1.11 & 0.30 & 1.17 & 0.35 & $1 \cdot 18$ & 0.29 & 0.038 \\
\hline TAG (mmol/l) & 1.74 & 0.97 & 1.90 & 1.31 & 1.80 & 1.30 & 0.50 & 1.81 & 1.45 & 1.80 & 0.96 & 1.80 & $1 \cdot 15$ & 0.54 \\
\hline Supplement use (\%) & & & & & & & 0.044 & & & & & & & $<0.001$ \\
\hline Medication use (\%)‡ & & & & & & & 0.29 & & & & & & & $<0.001$ \\
\hline
\end{tabular}

ADL, Activities of Daily Living.

Mean values were significantly different compared with the lowest quintile after Tukey's adjustment for multiple comparisons: ${ }^{*} P<0.05,{ }^{\star \star} P<0.01,{ }^{* \star *} P<0.001$.

† $P$ values for ANOVA for continuous variables across quintiles of total quantity and variety in fruit and vegetable intake were calculated by PROC GLM with SAS 9.2, respectively, and $\chi^{2}$ test for categorical variables across quintiles was performed by PROC FREQ.

$\ddagger$ Medication use was defined as yes if a participant had taken more than five types of medications within the past 12 months. 
Table 2. Cognitive performance by quintile of total quantity and variety in fruit and vegetable intake from the Boston Puerto Rican Health Study, 2004-9† (Mean values with their standard errors)

\begin{tabular}{|c|c|c|c|c|c|c|c|c|c|c|c|c|c|c|}
\hline & \multicolumn{6}{|c|}{ Fruit and vegetable intake } & \multicolumn{7}{|c|}{ Fruit and vegetable variety } & \multirow[b]{3}{*}{$P$ for trend $\neq$} \\
\hline & \multicolumn{2}{|c|}{ Quintile 1} & \multicolumn{2}{|c|}{ Quintile 3} & \multicolumn{2}{|c|}{ Quintile 5} & \multirow[b]{2}{*}{$P$ for trend $\ddagger$} & \multicolumn{2}{|c|}{ Quintile 1} & \multicolumn{2}{|c|}{ Quintile 3} & \multicolumn{2}{|c|}{ Quintile 5} & \\
\hline & Mean & SE & Mean & SE & Mean & SE & & Mean & SE & Mean & SE & Mean & SE & \\
\hline \multicolumn{15}{|l|}{ MMSE score } \\
\hline Model $1 \S$ & $23 \cdot 3$ & 0.2 & 23.4 & 0.2 & $23 \cdot 8$ & 0.2 & 0.13 & 23.1 & 0.2 & 23.4 & 0.2 & $24.0^{*}$ & 0.2 & $<0.001$ \\
\hline Model 2\| & 23.4 & 0.2 & $23 \cdot 3$ & 0.2 & $23 \cdot 7$ & 0.2 & 0.41 & $23 \cdot 2$ & 0.2 & 23.4 & 0.2 & 23.8 & 0.2 & 0.012 \\
\hline Model 3ף & 23.5 & 0.2 & $23 \cdot 3$ & 0.2 & $23 \cdot 7$ & 0.2 & 0.84 & $23 \cdot 2$ & 0.2 & $23 \cdot 4$ & 0.2 & $23 \cdot 8$ & 0.2 & 0.018 \\
\hline \multicolumn{15}{|c|}{ Executive function } \\
\hline Model $1 \S$ & -0.03 & 0.06 & -0.12 & 0.06 & $0 \cdot 10$ & 0.06 & 0.009 & -0.16 & 0.06 & -0.13 & 0.06 & $0.17^{\star \star *}$ & 0.06 & $<0.001$ \\
\hline Model 2\| & -0.04 & 0.07 & -0.19 & 0.07 & 0.03 & 0.07 & 0.076 & -0.19 & 0.07 & -0.13 & 0.07 & $0.07^{\star}$ & 0.07 & $<0.001$ \\
\hline Model 39 & 0.02 & 0.07 & -0.19 & 0.07 & -0.01 & 0.07 & 0.42 & -0.17 & 0.07 & -0.13 & 0.07 & $0.07^{\star}$ & 0.07 & $<0.001$ \\
\hline \multicolumn{15}{|l|}{ Memory } \\
\hline Model $1 \S$ & -0.16 & 0.07 & -0.05 & 0.07 & -0.001 & 0.07 & 0.99 & -0.24 & 0.07 & -0.06 & 0.07 & $0.07^{\star \star}$ & 0.07 & $<0.001$ \\
\hline Model 2॥ & -0.10 & 0.08 & -0.09 & 0.08 & -0.05 & 0.08 & 0.53 & -0.24 & 0.08 & -0.09 & 0.07 & 0.01 & 0.08 & 0.015 \\
\hline Model 3ी & -0.06 & 0.08 & -0.10 & 0.08 & -0.08 & 0.08 & 0.18 & -0.25 & 0.08 & -0.10 & 0.08 & $0.02^{*}$ & 0.08 & 0.007 \\
\hline \multicolumn{15}{|l|}{ Attention } \\
\hline Model $1 \S$ & 0.06 & 0.07 & 0.18 & 0.06 & 0.06 & 0.07 & 0.87 & -0.04 & 0.07 & 0.10 & 0.06 & $0.17^{\star}$ & 0.06 & 0.007 \\
\hline Model 2॥ & 0.08 & 0.08 & 0.17 & 0.07 & 0.04 & 0.08 & 0.60 & -0.04 & 0.08 & 0.10 & 0.07 & 0.16 & 0.07 & 0.022 \\
\hline Model 39 & 0.11 & 0.08 & 0.17 & 0.07 & 0.01 & 0.08 & 0.23 & -0.05 & 0.08 & 0.10 & 0.07 & 0.16 & 0.07 & 0.012 \\
\hline
\end{tabular}

MMSE, Mini Metal State Examination.

Mean values were significantly different compared with the lowest quintile after Tukey's adjustment for multiple comparisons: ${ }^{*} P<0.05,{ }^{\star *} P<0.01,{ }^{\star \star * *} P<0.001$.

tResults of the omitted quintiles generally follow the pattern of those of the shown quintiles, but are not presented for simplicity; adjusted means (with their standard errors) of cognitive performance scores according to quintiles of total quantity and variety in fruit and vegetable intake were calculated using general linear models (PROC GLM in SAS 9.2) after adjustment for covariates, respectively.

$\ddagger P$ values for trend were calculated using total quantity and variety in fruit and vegetable intake with PROC GLM in SAS 9.2, respectively.

§Model 1, adjusted for age (years), sex, educational attainment (none or primary school, secondary school, high school, or college or higher), household income below the threshold (yes/no), acculturation score (continuous), BMI $\left(\mathrm{kg} / \mathrm{m}^{2}\right)$ and total energy intake $(\mathrm{kJ} / \mathrm{d})$.

॥| Model 2, further adjusted for smoking status (never, past smoker or current smoker), alcohol use (never, moderate or high), physical activity score (continuous), supplement use (yes/no), taking more than five types of medications within the past 12 months (yes/no), activities of daily living scores (continuous), hypertension (yes/no) and diabetes (yes $/ \mathrm{no}$ ).

१ Model 3, further adjusted for fruit and vegetable variety for fruit and vegetable intake and vice versa. 


\section{Discussion}

To our knowledge, this is the first study to show that greater variety in fruit and vegetable intake was significantly associated with better cognitive function in middle-aged and older adults. Further, this association was maintained after adjustment for total quantity of fruit and vegetable intake.

Available studies about the association between fruit and vegetable intake and cognitive function are limited. In the Nurses' Health Study, intake of some types of vegetables, but neither of fruit, nor total fruit and vegetable intake, was associated with less cognitive decline after a 2 -year follow-up ${ }^{(8)}$. In the Chicago Health and Aging Project, with adults aged $\geq 65$ years, vegetable intake, but not citrus fruit intake, was associated with a slower rate of cognitive decline after a 6-year follow-up ${ }^{(9)}$. We did not observe significant associations between the total intake of fruit, the combination of fruit and vegetables and the MMSE score or individual cognitive domains (executive function, memory and attention). The total quantity of vegetable intake was significantly associated with the MMSE score only before adjustment for lifestyle factors, supplement and medication use, ADL score, diabetes and hypertension.

In contrast, higher variety in fruit and vegetable intake was associated with a better MMSE score, executive function, attention, memory function and several individual tests. These associations remained significant or continued to approach significance, even after adjustment for total quantity. The present results support the Dietary Guidelines for Americans, 2010 ${ }^{(32)}$ recommendation to increase variety in fruit and vegetable intake.

Fruit and vegetables are rich sources of antioxidant vitamins, B vitamins, minerals and other bio-compounds such as polyphenols. Our previous analysis has shown that higher variety in fruit and vegetable intake was significantly correlated with greater blood concentrations of carotenoids and ascorbic acid ${ }^{(24)}$. Oxidative stress has been indicated in the pathophysiological process of cognitive impairment and dementia $^{(33,34)}$. Antioxidants in fruit and vegetables may protect against neurodegeneration by scavenging free radicals. Nonetheless, previous studies have yielded inconsistent results about the associations between the intake of conventional antioxidant vitamins and cognitive decline and dementia $^{(16-19)}$. Limited data suggest that non-vitamin antioxidants may have higher neuroprotective capacity than antioxidant vitamins ${ }^{(35)}$. Further, different fruits and vegetables have varied bioactive components. Therefore, the combination of multiple types of fruits and vegetables may have additive and/or synergistic effects on physiology above that of isolated nutrients or individual foods. Wang et al. ${ }^{(36)}$ recently demonstrated that there were additive or synergistic effects on the total antioxidant capacity of the combination of specific foods within food categories (fruit or vegetable). More importantly, the combination of specific foods across categories was more likely to have synergistic effects on the total antioxidant capacity than the combinations within categories ${ }^{(36)}$. Their data support our present findings that higher variety in fruit and vegetable intake, relative to total quantity, was more strongly associated with better cognitive function. 
The observed associations between higher variety in fruit and vegetable intake and cognitive function could also be due to other nutrients and/or bio-compounds. For example, low folate concentrations have been observed among those with neurodegenerative disorders ${ }^{(37)}$. A higher dietary folate intake has been linked with a slower decline of cognitive function $^{(38)}$. In addition, inflammatory factors have been associated with poor cognitive function, cognitive impairment and dementia ${ }^{(39)}$. Higher variety in fruit and vegetable intake was also associated with lower C-reactive protein (a chronic low-grade inflammatory biomarker) in our previous analysis ${ }^{(24)}$.

Strengths of the present study include a large sample size and our ability to adjust for a variety of covariates. We were able to examine the independent associations between variety in fruit and vegetable intake and cognitive function because the correlation between total quantity and variety in fruit and vegetable intake was moderate. We administrated a series of cognitive tests to examine several domains of cognition. However, due to the cross-sectional nature of the present analysis, we cannot infer a casual direction to our observed association. It is possible that those with lower cognitive function may change their dietary intake, or may recall their dietary intake in a less accurate way than others. However, we have previously shown that interviewer-administrated FFQ reporting may be valid in those with cognitive impairment, due to the superior retention of generic memory, relative to episodic memory for specific events, in most individuals ${ }^{(40)}$. Additionally, residual confounding remains a possibility. While our sample is restricted to the Puerto Rican population, there is no reason to suspect that the present results cannot be generalised to other populations or minority groups.

In conclusion, greater variety in fruit and vegetable intake was associated with better cognitive function in middle-aged and older adults. An emphasis on increasing variety, rather than only quantity of fruits and vegetables, may be advisable for this and other populations, to delay or prevent the onset of cognitive impairment and related chronic diseases.

\section{Acknowledgements}

This study was supported by the National Institute on Aging of the National Institutes of Health (NIH) (P01AG023394, R01AG02708), the National Heart Lung and Blood Institute of NIH (P50HL105185), and the US Department of Agriculture Agricultural Research Service (contract no. 58-1950-7-707). X. Y. contributed to the data analysis, the interpretation of the data and the writing of the manuscript. S. N. B. and K. L. T. contributed to the interpretation of the data and the critical revision of the manuscript. K. L. T. obtained the funding, conceived the study and supervised the data collection. All authors read and approved the final manuscript. None of the authors had any conflicts of interest.

Supplementary tables are available online at http://www. journals.cambridge.org/bjn

\section{References}

1. Dauchet L, Amouyel P, Hercberg S, et al. (2006) Fruit and vegetable consumption and risk of coronary heart disease: a meta-analysis of cohort studies. J Nutr 136, 2588-2593.
2. He FJ, Nowson CA, Lucas M, et al. (2007) Increased consumption of fruit and vegetables is related to a reduced risk of coronary heart disease: meta-analysis of cohort studies. J Hum Hypertens 21, 717-728.

3. Dauchet L, Amouyel P \& Dallongeville J (2005) Fruit and vegetable consumption and risk of stroke: a meta-analysis of cohort studies. Neurology 65, 1193-1197.

4. He FJ, Nowson CA \& MacGregor GA (2006) Fruit and vegetable consumption and stroke: meta-analysis of cohort studies. Lancet 367, 320-326.

5. Koushik A, Hunter DJ, Spiegelman D, et al. (2007) Fruits, vegetables, and colon cancer risk in a pooled analysis of 14 cohort studies. J Natl Cancer Inst 99, 1471-1483.

6. Lunet N, Valbuena C, Vieira AL, et al. (2007) Fruit and vegetable consumption and gastric cancer by location and histological type: case-control and meta-analysis. Eur J Cancer Prev 16, 312-327.

7. Pavia M, Pileggi C, Nobile CG, et al. (2006) Association between fruit and vegetable consumption and oral cancer: a meta-analysis of observational studies. Am J Clin Nutr 83, 1126-1134.

8. Kang JH, Ascherio A \& Grodstein F (2005) Fruit and vegetable consumption and cognitive decline in aging women. Ann Neurol 57, 713-720.

9. Morris MC, Evans DA, Tangney CC, et al. (2006) Associations of vegetable and fruit consumption with age-related cognitive change. Neurology 67, 1370-1376.

10. Sabia S, Nabi H, Kivimaki M, et al. (2009) Health behaviors from early to late midlife as predictors of cognitive function: the Whitehall II study. Am J Epidemiol 170, 428-437.

11. Hughes TF, Andel R, Small BJ, et al. (2010) Midlife fruit and vegetable consumption and risk of dementia in later life in Swedish twins. Am J Geriatr Psychiatry 18, 413-420.

12. Engelhart MJ, Geerlings MI, Ruitenberg A, et al. (2002) Dietary intake of antioxidants and risk of Alzheimer disease. JAMA 287, 3223-3229.

13. Morris MC, Evans DA, Bienias JL, et al. (2002) Dietary intake of antioxidant nutrients and the risk of incident Alzheimer disease in a biracial community study. JAMA 287, 3230-3237.

14. Laurin D, Masaki KH, Foley DJ, et al. (2004) Midlife dietary intake of antioxidants and risk of late-life incident dementia: the Honolulu-Asia Aging Study. Am J Epidemiol 159, 959-967.

15. Luchsinger JA, Tang MX, Shea S, et al. (2003) Antioxidant vitamin intake and risk of Alzheimer disease. Arch Neurol 60, 203-208.

16. Yaffe K, Clemons TE, McBee WL, et al. (2004) Impact of antioxidants, zinc, and copper on cognition in the elderly: a randomized, controlled trial. Neurology 63, 1705-1707.

17. Petersen RC, Thomas RG, Grundman M, et al. (2005) Vitamin $\mathrm{E}$ and donepezil for the treatment of mild cognitive impairment. N Engl J Med 352, 2379-2388.

18. Kang JH, Cook NR, Manson JE, et al. (2009) Vitamin E, vitamin $\mathrm{C}$, beta carotene, and cognitive function among women with or at risk of cardiovascular disease: the Women's Antioxidant and Cardiovascular Study. Circulation 119, 2772-2780.

19. Grodstein F, Kang JH, Glynn RJ, et al. (2007) A randomized trial of beta carotene supplementation and cognitive function in men: the Physicians' Health Study II. Arch Intern Med 167, 2184-2190.

20. Liu RH (2003) Health benefits of fruit and vegetables are from additive and synergistic combinations of phytochemicals. Am J Clin Nutr 78, 517S-520S. 
21. Tucker K, Mattei J, Noel S, et al. (2010) The Boston Puerto Rican Health Study, a longitudinal cohort study on health disparities in Puerto Rican adults: challenges and opportunities. BMC Public Health 10, 107.

22. Mungas D (1991) In-office mental status testing: a practical guide. Geriatrics 46, 54-58, 66.

23. Cook AJ \& Friday JE (2004) Pyramid servings database for USDA survey food codes. Version 2.0. Beltsville, MD: US Department of Agriculture, Agricultural Research Service, Community Nutrition Research Group. http://www.ars usda.gov/Services/docs.htm?docid=8634.

24. Bhupathiraju SN \& Tucker KL (2011) Greater variety in fruit and vegetable intake is associated with lower inflammation in Puerto Rican adults. Am J Clin Nutr 93, 37-46.

25. Folstein MF, Folstein SE \& McHugh PR (1975) "Mini-mental state". A practical method for grading the cognitive state of patients for the clinician. J Psychiatr Res 12, 189-198.

26. Artiola Fortuny L, Hermosillo Romo D, Heaton RK, et al. (2000) Manual de Normas y Procedimientos para la Batería Neuropsicológica en Español (Standards and Procedures Manual for Neuropsychological Battery in Spanish). Lisse: Swets \& Zeitlinger.

27. Wolf-Klein GP, Silverstone FA, Levy AP, et al. (1989) Screening for Alzheimer's disease by clock drawing. J Am Geriatr Soc 37, 730-734.

28. Beery K (1989) The Developmental Test of Visual-Motor Integration Manual, revised ed. Cleveland: Modern Curriculum Press.

29. Department of Health and Human Services (2007) Annual update of the HHS poverty guidelines. Federal Register $\mathbf{7 2}$, 3147-3148.

30. Tucker KL, Falcon LM, Bianchi LA, et al. (2000) Self-reported prevalence and health correlates of functional limitation among Massachusetts elderly Puerto Ricans, Dominicans, and non-hispanic white neighborhood comparison group. J Gerontol A Biol Sci Med Sci 55, M90-M97.
31. American Diabetes Association (2008) Diagnosis and classification of diabetes mellitus. Diabetes Care 31, Suppl. $1, \mathrm{~S} 55-\mathrm{S} 60$.

32. U.S. Department of Agriculture and U.S. Department of Health and Human Services (2010) Dietary Guidelines for Americans, 2010. Washington, DC: U.S. Government Printing Office.

33. Pratico D, Clark CM, Liun F, et al. (2002) Increase of brain oxidative stress in mild cognitive impairment: a possible predictor of Alzheimer disease. Arch Neurol 59, 972-976.

34. Lovell MA \& Markesbery WR (2007) Oxidative damage in mild cognitive impairment and early Alzheimer's disease. J Neurosci Res 85, 3036-3040.

35. Dai Q, Borenstein AR, Wu Y, et al. (2006) Fruit and vegetable juices and Alzheimer's disease: the Kame Project. Am J Med 119, 751-759.

36. Wang S, Meckling KA, Marcone MF, et al. (2011) Synergistic, additive, and antagonistic effects of food mixtures on total antioxidant capacities. J Agric Food Chem 59, 960-968.

37. Mattson MP \& Shea TB (2003) Folate and homocysteine metabolism in neural plasticity and neurodegenerative disorders. Trends Neurosci 26, 137-146.

38. Tucker KL, Qiao N, Scott T, et al. (2005) High homocysteine and low $\mathrm{B}$ vitamins predict cognitive decline in aging men: the Veterans Affairs Normative Aging Study. Am J Clin Nutr 82, 627-635.

39. Komulainen P, Lakka TA, Kivipelto M, et al. (2007) Serum high sensitivity $\mathrm{C}$-reactive protein and cognitive function in elderly women. Age Ageing 36, 443-448.

40. Arsenault LN, Matthan N, Scott TM, et al. (2009) Validity of estimated dietary eicosapentaenoic acid and docosahexaenoic acid intakes determined by interviewer-administered food frequency questionnaire among older adults with mild-to-moderate cognitive impairment or dementia. Am J Epidemiol 170, 95-103. 